STATE OF ILLINOIS

DIVIGHT H. GREEN, Governor.

DEPARTMENT OF REGISTRATION AND EDUCATION

FRANK G. THOMPSON, Director

DIVISION OF THE

STATE GEOLOGICAL SURVEY

M. M. LEIGHTON, Chief

URBANA

REPORT OF INVESTIGATIONS-NO. 98

SMALLER FORAMINIFERA FROM THE PORTERS CREEK FORMATION (PALEOCENE) OF ILLINOIS

BY

CHALMER L. COOPER

REPRINTEd From the Journal of Paleontology, Vol. 18, No. 4, July, 1944

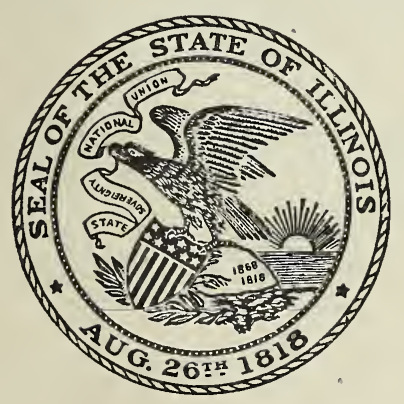

PRINTED BY AUTHORITY OF THE STATE OF ILLINOIS

URBANA, ILLINOIS 


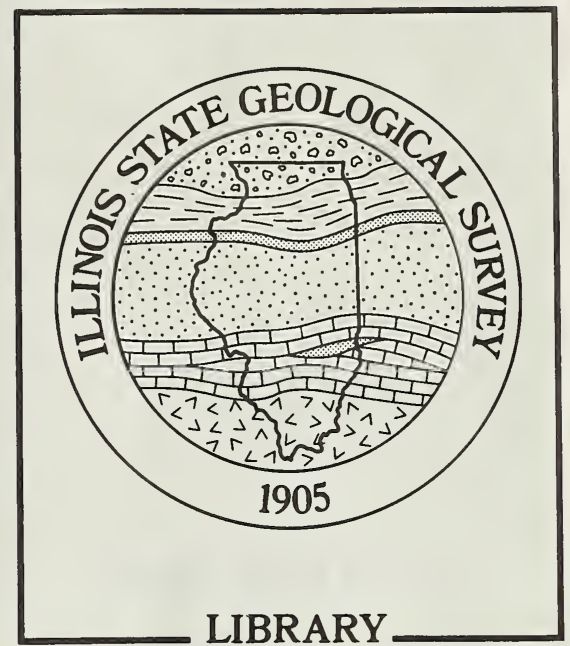

STATE OF ILLINOIS

HON. DWIGHT H. GREEN, Governor

DEPARTMENT OF REGISTRATION AND EDUCATION HON. FRANK G. THOMPSON, Director

\section{BOARD OF NATURAL RESOURCES AND CONSERVATION}

HON. FRANK G. THOMPSON, Chairman

EDSON S. BASTIN, Pr.D., D.Sc., Geology

ROGER ADAMS, PH.D., D.Sc., Chemistry

LOUIS R. HOWSON, C.E., Engineering

WILLIAM TRELEASE, D.Sc., LL.D., Biology

EZRA JACOB KRAUS, PH.D., D.Sc., Forestry

ARTHUR CUTTS WILLARD, D.ENGR., LL.D

President of the University of Illinois

GEOLOGICAL SURVEY DIVISION

M. M. LEIGHTON, Chief 


\section{STATE GEOLOGICAL SURVEY DIVISION}

\section{Natural Resources Building, Urbana}

M. M. LEIGHTON, Ph.D., Chief

Enid Townley, M.S., Assistant to the Chief

VElda A. MILlaRd, Junior Asst. to the Chief

Helen E. McMorris, Secretary to the Chief

Betty J. IVesterbeék, Geological Assistant

\section{GEOLOGICAL RESOURCES}

Coal

G. H. CADy, PH.D., Senior Geologist and Head

L. C. MCCABE, PH.D., Geologist (on leave)

R. J. Helfinstine, M̈.S., Mech. Engineer

Charles C. Boley, M.S., Assoc. Mining Eng.

Heinz A. Lowenstam, Pri.D., Assoc. Geologist

Bryan Parks, M.S., Asst. Geologist

EARLE F. TAYlor, M..S., Asst. Geologist (on leave)

RALPH F. STrete, A.M., Asst. Geologist

M. W. Pullen, Jr., M.S., Asst. Geologist

RoberT M. KoSa KE, M.A., Asst. Geologist

Robert Ellingwood, B.S., Asst. Geologist

GEORGE M. Wilson, B.S.,' Asst. Geologist

Ar Nold EDDInGS, B.A.,' Research Assistant (on leave)

Henry L. Smith, A.B., Research Assistant

Raymond Siever, B.S., Research Assistant

John A. Harrison, B.S., Research Assistant (on leave)

Mary E. Barnes, B.S., Research Assistant

Robert T. Anderson, M.A., Asst. Physicist

Margaret Parker, B.S., Research Assistant

Industrial Minerals

J. E. Lamar, B.S., Geologist and Head

H. B. Willman, Ph.D., Assoc. Geologist

Robert M. Grogan, Ph.D.,. Assoc. Geologist

ROBERT R. REYNOLDS, M.S., Asst Geologist

Margaret Copeland, A.B., Research Assistant

Oil and Gas

A. H. Bell, Pr.D., Geologist and Head

CARl A. BAYs, Ph.D., Geologist and Engineer

Frederick SQuires, B.S., Petroleum Engineer

Stewart FolK, M.S., Assoc. Geologist

William H. Easton, Ph.D., Assoc. Geologist

ERnest P. Du Bors, Ph.D., Asst. Geologist

Paul G. LuCKhardt, M.S., Asst. Geologist (on leave)

Wayne F, Meents, Asst. Geologist

JAM ES S. Yolton, M.S.. Asst. Geologist

Margaret Sands, B.S., Research Assistant

Robert F. Smiti, A.B., Research Assistant

Areal and Engineering Geology

George E. Exblaw, Ph.D., Geologist and Head

Richard F. Fish ER, M.S., Asst. Geologist

Subsurface Geology

L. E. Workman, M.S., Geologist and Head CARl A. Bays, Ph.D., Geologist and Engineer Charles W. Carter, Ph.D., Assoc. Geologist Robert R. Storm, A.B., Assoc. Geologist

Arnold C. Mason, B.S., Assoc. Geologist (on leave)

C. Leland Horberg, Ph.D., Assoc. Geologist

Fran K E. Tippie, B.S., Asst. Geologist

Merlyn B. Buhle, M.S., Asst. Geologist

Paul Herbert, Jr., B.S., Asst. Geologist

Charles G. Joll n son, A.B., Asst. Geologist (on leave)

Dorothy B. Speziale, M.S., Asst. Geologist

MarVI N P. MEYER, B.S., Asst. Geologist

Margaret CAStle, Research Assistant

Ruth E. Roth, B.S., Research Assistant
Stratigraphy and Paleontology

J. Marvin Weller, Ph.D., Geologist and Head

Chalm Er L. CoOPER, M.S., Assoc. Geologist.

William H. Easton, Ph.D., Assoc. Geologist

\section{Petrography}

Ralph E. Grim, Ph.D., Petrographer

Richards A. Rowland, Ph.D., Asst. Petrographer (on leave)

Physics

R. J. Piersol, Ph.D., Physicist

B. J. GreENWOOD, B.S., Mech. Engineer

Donald O. Holland, M.S., Asst. Physicist (on leave)

\section{GEOCHEMISTRY}

Frank H. ReEd, Pri.D., Chief Chemist

H. W. JACKMAN, M.S.E., Chemical Engineer

James C. McCullough, Research Associate

Elizabet Ross Mills, M.S., Research Assistant

Coal

G. R. Yоне, Pн.D., Chemist

Herman S. Levine, B.S., Research Assistant

Industrial Minerals

J. S. MAChin, Ph.D., Chemist and Head

Delbert L. Hanna, A.M., Asst. Chemist

\section{Fluorspar}

G. C. Finger, Ph.D., Chemist

$X$-ray and Spectrography

W. F. Bradley, Ph.D., Chemist

Analytical

O. W. Rees, Pr.D., Chemist and Head Howard S. Clar K, A.B., Assoc. Chemist L. D. MCVICKER, B.S., Chemist

P. W Henline, M.S., Assoc. Chemical Engineer William F. Wagner, M.S., Asst. Chemist

CAM ERON D. LewIS, B.A., Asst. Chemist

Herbert N. HazelKorn, B.S., Research Assistant

William T. ABEL, B.A., Rescarch Assistant

CAROL J. ADAMS, B.S., Research Assistant

\section{MINERAL ECONOMICS}

W. H. Voskuil, Pн.D., Mineral Economist Douglas F. Stevens, M.E., Research Associate

ETHEL M. KING, Research Assistant

\section{PUBLICATIONS AND RECORDS}

George E. Ekblaw, Ph.D., Geologic Editor

ChalMER L. COOPER, M.S., Geologic Editor

DOROTHY E. Rose, B.S., Technical Editor

Portia ALLYN SMith, Technical Files Clerk

ROSEMARY METZGer, Technical Assistant

MEREDITH M. CalKins, Geologic Draftsman

Beulah Featherstone, B.F.A., Asst. Geologic Draftsman

Leslie D. Vaugiran, Asst. Photographer

Consultants: Ceramics, Cullen W. Parmelee, M.S., D.Sc., and RalPh K. Hursh, B.S., University of Illinois Mechanical Engineering, SeICHI Konzo, M.S., University of Illinois

Topographic Mapping in Cooperation with the United States Geological Survey.

This report is a Contribution of the Stratigraphy and Paleontology Section. 
Digitized by the Internet Archive in 2012 with funding from University of Illinois Urbana-Champaign 


\title{
SMALLER FORAMINIFERA FROM THE PORTERS CREEK FORMATION (PALEOCENE) OF ILLINOIS
}

\author{
CHALMER L. COOPER \\ Illinois State Geological Survey, Urbana ${ }^{1}$
}

\begin{abstract}
A microfauna of smaller Foraminifera from the cuttings of a water well in Cache, southern Alexander County, shows the occurrence of Lower Eocene (Paleocene) sediments almost to the northern limit of the Mississippi embayment. Six families are represented, comprising 15 genera and 30 species. More than half of the species belong to the Lagenidae, with two to four species in each of the Heterohelicidae, Ellipsoidinidae, Rotaliidae, Globigerinidae, and Anomalinidae. Two new species are described.
\end{abstract}

\section{INTRODUCTION}

$\mathrm{T}$ $\checkmark$ HE ORIGINAL northern limit of Tertiary sediments in the Mississippi embayment is unknown. The Cretaceous deposits in the Mississippi Valley probably extended as far north as southeastern Minnesota (Sardeson, 1898) and may have been at one time coextensive with those of the Midcontinent region. To a certain extent the succeeding Paleocene sea flooded the same areas. Therefore it is probable that the earliest Cenozoic formations originally extended farther north than the present outcrops in southern Illinois would indicate. On the other hand, shore line conditions are indicated by the absence of some marine horizons which are present farther south. In Tennessee the Ripley group is composed of the McNairy sand over- and underlain by the Owl Creek and Coon Creek marine formations. The last two formations are not recognized in Kentucky and from the very sandy and glauconitic character of the Ripley in Illinois it is possible that only the McNairy is present here also. The possibility should be pointed out that the shoreward nonfossiliferous phases of these higher and lower formations would take on the sandy characteristics of the McNairy and would therefore be indistinguishable from it.

The same is true of the Wilcox group. In Tennessee it is composed of the Holly Springs sandy formation which is over-and underlain by the Grenada and Ackermann marine shaly and limy formations. The latter is not recognized in Kentucky and from

${ }^{1}$ Published with permission of the Chief. the sandy character of the Wilcox at the northern limit of its outcrop the Grenada is probably also absent. This would leave the Holly Springs as the sole representative of the Wilcox group at the northern end of the embayment, although the possible presence of the sandy shoreward phase of the other members of the group must not be overlooked.

The northernmost outcrop of Paleocene formations shown on the geologic map of the United States (Stose, 1932) is found on the eastern side of the Mississippi embayment just south of the Kentucky line in the north-central part of Henry County, Tennessee. On the west side of the embayment the most northern extent of the Midway is shown southeast of Newport in northeastern White and southeastern Independence counties, Arkansas. However, Lamar and Sutton (1930, fig. 1) mapped the Porters Creek formation around the eastern and northern edges of the embayment across Kentucky and into Alexander County, Illinois, as far west as the town of Unity where the formation dips beneath younger sediments. They also mentioned (p. 953) the possible occurrence of the formation at Idalia and Bloomfield, central Stoddard County, Missouri. However, no correlation of the Porters Creek of Illinois, other than with the formation in Tennessee, was made by these writers. Subsequent work has confirmed the suspected occurrences and revealed others in Missouri (Farrar and McManamy, 1937; Stewart, McManamy, and McQueen, 1943) and has shown that a number of changes are necessary in the Illinois portion of the map (see text fig. 1). 


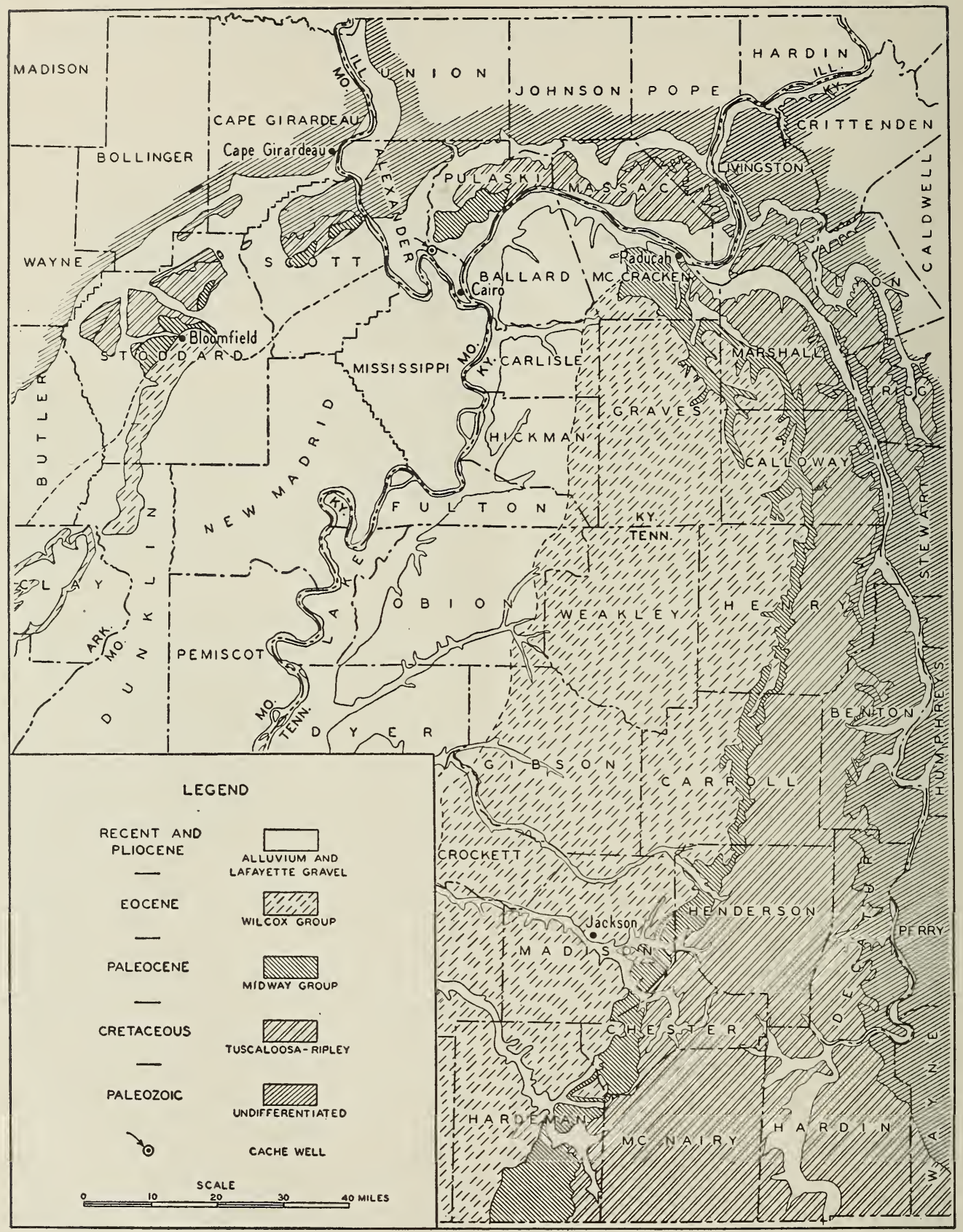

FIG. 1.-Map of the upper part of the Mississippi embayment area showing the outcrop of the Wilcox and Midway groups and the location of the well at Cache, Alexander County, Illinois.

From geologic maps of Tennessee (1923), Kentucky (1929), Illinois (1939), and Missouri (1939, revised by recent work of the Missouri Geological Survey). 
The formation containing the fossils described was named the "Porters Creek group" for exposures on Porters Creek, Hardemann County, Tennessee, by Stafford $(1864$, p. 368) where it has a thickness of 200 to 300 feet. There it overlies the Clayton formation, which together with the Porters Creek, comprises the Midway group of the central and northern embayment areas and underlies the Ackerman formation of Wilcox age. Harris (1896) first correlated the formation with the Midway.

The Cretaceous and Tertiary sediments in the northern part of the embayment in Illinois and Missouri are almost completely devoid of calcareous material and fossil remains, except for an occasional fossil cast. Lamar and Sutton (1930, p. 848) believe the original calcareous material, while once present in the sediments, has been removed by leaching.

The microfauna described was obtained from the cuttings of a water well drilled in 1938 at Cache, Illinois, in $\mathrm{SE}_{\frac{1}{4}} \mathrm{SE}_{\frac{1}{4}} \mathrm{SW}_{\frac{1}{4}}$ sec. 19, T. 16 S., R. 1 W., Alexander County. The well was drilled for O. R. Bourland by E. M. Gould, who furnished a driller's $\log$ and 5 -foot samples of the drill cuttings to the Subsurface Division of the Illinois State Geological Survey, where they were studied and described by Earl C. Cochrum. It was Mr. Cochrum who called my attention to the occurrence of the numerous Foraminifera found in the samples from a depth of 115 to 135 feet. All but four of the species described came from the two sam.

Correlation of Post-Paleozoic Sediments in the Upper Mississippi Embayment Area

\begin{tabular}{|c|c|c|c|c|c|c|}
\hline AGE & ARKANSAS & & TENNESSEE ${ }^{1}$ & KENTUCKY ${ }^{2}$ & ILLINOIS & MISSOURI $^{3}$ \\
\hline $\begin{array}{c}\text { RECENT } \\
\text { AND/OR } \\
\text { PLEISTOCENE }\end{array}$ & $\begin{array}{l}\text { Alluvium } \\
\text { and } \\
\text { Loess }\end{array}$ & \multicolumn{2}{|r|}{$\begin{array}{l}\text { Alluvium } \\
\text { and } \\
\text { Loess }\end{array}$} & $\begin{array}{l}\text { Alluvium } \\
\text { and } \\
\text { Loess }\end{array}$ & $\begin{array}{l}\text { and } \\
\text { Loess }\end{array}$ & $\begin{array}{l}\text { Alluvium } \\
\text { and } \\
\text { Loess }\end{array}$ \\
\hline PLIOCENE & Gravel & \multicolumn{2}{|c|}{ Gravel } & Lafayette & "Lafayette" & Gravel \\
\hline \multirow{4}{*}{ EOCENE } & Jackson & \multicolumn{2}{|c|}{ Jackson } & Jackson & & \\
\hline & Claiborne & & & & & \\
\hline & Wilcox & 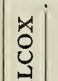 & $\begin{array}{l}\text { Grenada } \\
\text { Holly Springs }\end{array}$ & $\begin{array}{l}\text { Grenada } \\
\text { Holly Springs }\end{array}$ & Wilcox & Wilcox \\
\hline & & & Ackerman & & & \\
\hline & & 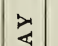 & Porters Creek & Porters Creek & Porters Creek & Porters Creek \\
\hline & Midway & 窇 & Clayton & & Clayton & Clayton \\
\hline CRET & Nacatoch & 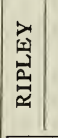 & $\begin{array}{l}\text { Owl Creek } \\
\text { McNairy } \\
\text { Coon Creek }\end{array}$ & McNairy & 盍 McNairy & \begin{tabular}{l|l} 
Owl Creek & McNairy \\
& McNoon \\
Coon & Creek?
\end{tabular} \\
\hline CNE HACEVOS & & Sel & $\operatorname{lma}$ & Selma & & Selma (?) \\
\hline & & $\mathrm{Eu}$ & Itaw & Eutaw & & Eutaw (?) \\
\hline & & $\mathrm{Tu}$ & iscaloosa & Tuscaloosa & & \\
\hline
\end{tabular}

1 Whitlatch (1940);

2 McFarlan (1943);

Kansas Geol. Soc. (1939) and Stewart, McManamy, and McQueen (1943). 
ples at 120 to 130 feet in depth. A graphic section based on the sample study is shown in text figure 2. The map (fig. 1) is based on the published state geologic maps of Tennessee (1923), Kentucky (1929), Arkansas (1929) and Missouri (1939) and the preliminary edition of the new geologic map of

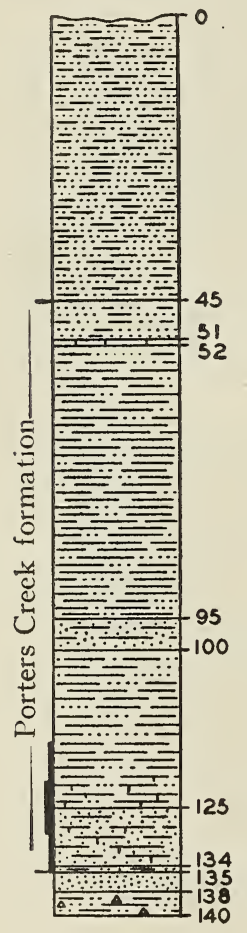

Alluvium

Siltstone, yellowish-brown, fine-grained

Limestone, brownish-gray, fine-grained

Shale, silty, brownish-gray, flaky

Shale, silty, micaceous, glauconitic, flaky

Shale, silty, micaceous, flaky, calcareous at base

Shale, silty, calcareous, micaceous, glauconitic

Glauconite, dark green, slightly calcareous

Sandstone, water-bearing

Sand, cherty, silty, glauconitic, fine to coarse

Fig. 2.-Graphic section showing the beds encountered in the Cache well and the fossiliferous zone indicated by the heavy border at the left margin.

Illinois (Weller, 1939), with some modifications in Missouri. Mr. J. E. Lamar contributed to the stratigraphic discussion.

\section{FAUNAL DISCUSSION}

The fauna from the Cache well consists of 30 species of the smaller Foraminifera, of which more than half (16 species) belong to the family Lagenidae. This is in accord with conclusions based on studies of Eocene foraminifera by Plummer (1927, p. 12), Cushman (1940), and Toulmin (1941). The other families represented are: Ellipsoidinidae and Anomalinidae, 4 species each; and Heterochilicidae, Rotaliidae, and Globigerinidae, 2 species each. Fifteen genera are represented.

The fauna is distinct from that in the Upper Cretaceous, which can be recognized by its preponderance of Textulariidae and, though very much like that of the Wilcox above, lacks the frequent occurrence of many species of the Miliolidae which characterizes the younger group.

\section{SYSTEMATIC DESCRIPTIONS \\ Family Lagenidae Cushman \\ Genus Chrysaligonium Schubert \\ Chrysaligonium granti (Plummer) \\ Plate 54, figures 26, 27}

Nodosaria filiformis Carsey, 1926, Texas Univ. Bull. 2612, p. 33, vol. 7, fig. 8; Upper Cretaceous, Texas.

Nodosaria granti Plummer, 1927, Texas Univ. Bull. 2644, p. 83, pl. 5, figs. 9a-d; Midway group, Paleocene, Texas.

Dentalina granti, Plummer, 1931, Texas Univ. Bull. 3101, p. 149, pl. 11, figs. 8, 9; Upper Cretaceous, Texas.-Jennings, 1936, Bull. Am. Paleontology, vol. 23, p. 176, pl. 29, fig. 6; Upper Cretaceous, New Jersey.

Ellipsonodosaria granti, ? Cushman, 1936, Cushman Lab. Foram. Research Contr., vol. 2, p. 151, pl. 9, figs. 3-5; Upper Cretaceous, Texas and Arkansas.-Cushman, 1940, idem, vol. 16, p. 69 , pl. 12, fig. 3; Midway group, Paleocene, Alabama.

Chrysaligonium granti, Toulmin, 1941, Jour. Paleontology, vol. 15, p. 589, pl. 79, figs. 34, 35; Salt Mountain limestone, Eocene, Alabama.

Test elongate, slender, slightly arcuate; chambers elongate, cylindrical; wall thick, smooth; suture flush with surface. Diameter of central chamber, $0.16 \mathrm{~mm}$.; length about $0.28 \mathrm{~mm}$.

This form, even though the species is somewhat variable, shows the general characteristics. The fragment (pl. 54, fig. 27) shows the apical spine. Its presence in Upper Cretaceous and lower Wilcox beds make it valueless as an index.

Genus Dentalina d'Orbigny

Dentalina cf. D. Communis (d'Orbigny)

Plate 55, figure 10

Nodosaria (Dentalina) communis d'Orbigny, 1826, Soc. Nat. Sci. Ann., vol. 7, p. 254, no. 35.

Nodosaria communis, Cushman, 1923, U. S. Nat. Mus. Bull. 104 , pt. 4 , p. 75 ; pl. 12 , figs. 3, 4; Recent, Atlanțic. 
Faunal List and Geologic Range

\begin{tabular}{|c|c|c|}
\hline Species & Pl. fig. & Previous known range \\
\hline \multirow{15}{*}{$\begin{array}{l}\quad \text { LAGENIDAE } \\
\text { Chrysaligonium granti (Plummer) } \\
\text { Dentalina cf. D. communis (d'Orbigny) } \\
\text { Dentalina cf. D. cooperensis Cushman } \\
\text { Dentalina pauperata d'Orbigny } \\
\text { Dentalina spira Cooper, n. sp. } \\
\text { Lagena laevis (Montagu) } \\
\text { Nodosaria latejugata Gümbel } \\
\text { Nodosaria spinocostata Cooper, n. } \mathrm{sp} \text {. } \\
\text { Nodosaria cf. } N \text {. vertebralis (Batsch) } \\
\text { Nodosaria cf. N. zippei Reuss } \\
\text { Palmula meglameryae Toulmin } \\
\text { Robulus inornatus (d'Orbigny) } \\
\text { Robulus magnificus Toulmin } \\
\text { Robulus midwayensis (Plummer) } \\
\text { Vaginulina gracilis Plummer } \\
\text { Vaginulina robusta Plummer }\end{array}$} & \multirow{15}{*}{$\begin{array}{l}54 ; 16,27 \\
55 ; 20 \\
54 ; 21 \\
54 ; 10 \\
54 ; 1,2 \\
54 ; 6,7 \\
55 ; 24,25 \\
54 ; 11 \\
55 ; 14,15 \\
55 ; 18,19 \\
55 ; 20,21 \\
55 ; 26,27 \\
55 ; 1,2 \\
55 ; 22,23 \\
55 ; 5,6 \\
55 ; 16,17\end{array}$} & \multirow{5}{*}{$\begin{array}{l}\text { Upper Cretaceous-Eocene } \\
\text { Eocene-Recent } \\
\text { Eocene } \\
\text { Jurassic-Recent? }\end{array}$} \\
\hline & & \\
\hline & & \\
\hline & & \\
\hline & & \\
\hline & & \multirow{3}{*}{$\begin{array}{l}\text { Eocene-Recent } \\
\text { Eocene-Oligocene }\end{array}$} \\
\hline & & \\
\hline & & \\
\hline & & \multirow{2}{*}{$\begin{array}{l}\text { Cretaceous-Recent } \\
\text { Cretaceous }\end{array}$} \\
\hline & & \\
\hline & & \multirow{2}{*}{$\begin{array}{l}\text { Paleocene-Eocene } \\
\text { Eocene-Miocene }\end{array}$} \\
\hline & & \\
\hline & & $\begin{array}{l}\text { Eocene } \\
\text { Paleocene-Eocene }\end{array}$ \\
\hline & & $\begin{array}{l}\text { Paleocene-Eocene } \\
\text { Paleocene }\end{array}$ \\
\hline & & \\
\hline \\
\hline $\begin{array}{l}\text { Rectogïmbelina alabamensis Cushman } \\
\text { Siphonogeneroides? eleganta (Plummer) }\end{array}$ & $\begin{array}{l}55 ; 3,4 \\
54 ; 14\end{array}$ & $\begin{array}{l}\text { Paleocene } \\
\text { Paleocene }\end{array}$ \\
\hline \multicolumn{3}{|l|}{ ELLIPSOIDINIDAE } \\
\hline Ellipsonodosaria lepidula (Schwager) & $54 ; 23$ & \multirow{4}{*}{$\begin{array}{l}\text { Upper Cretaceous-Pliocene } \\
\text { Paleocene } \\
\text { Paleocene-Pliocene } \\
\text { Paleocene }\end{array}$} \\
\hline Ellipsonodosaria plummerae Cushman & $54 ; 18,19$ & \\
\hline Ellipsonodasaria sagrinensis (Bagg) & $54 ; 22$ & \\
\hline Ellipsonodosaria spinulosa (Plummer) & $54 ; 20$ & \\
\hline \multicolumn{3}{|l|}{ ROTALIIDAE } \\
\hline Eponides exigua limbata (Plummer) & $55 ; 11-13$ & \multirow{2}{*}{$\begin{array}{l}\text { Paleocene } \\
\text { Cretaceous-Paleocene }\end{array}$} \\
\hline Siphonina prima Plummer & $55 ; 7-9$ & \\
\hline \multicolumn{3}{|l|}{ GLOBIGERINIDAE } \\
\hline Globigerina compressa Plummer & $54 ; 8,9$ & Paleocene-Eocene \\
\hline Globigerina triloculinoides Plummer & $54 ; 12,13$ & Paleocene-Eocene \\
\hline \multicolumn{3}{|l|}{ ANOMALINIDAE } \\
\hline Anomalina acuta Plummer & $54 ; 3-5$ & Paleocene-Eocene \\
\hline Anomalina midwayensis (Plummer) & $54 ; 15-17$ & Paleocene \\
\hline $\begin{array}{l}\text { Cibicides alleni (Plummer) } \\
\text { Cibicides vulgaris (Plummer) }\end{array}$ & $\begin{array}{l}54 ; 24,25 \\
54 ; 28,29\end{array}$ & Cretaceous-Paleocene \\
\hline
\end{tabular}

Dentalina communis, Howe and Wallace, 1932 , Louisiana Dept. Cons. Geol. Bull. 2, p. 24, pl. 6, fig. 8; Upper Eocene, Louisiana.?Cushman and Garrett, 1932, Cushman Lab. Foram. Research Contr., vol. 8, p. 55, pl. 7, figs. 12, 13; Wilcox group, Eocene, Alabama.?Cushman and Garrett, 1939, Cushman Lab. Foram. Research Contr., vol. 15 , p. 80 , pl. 14 , figs. 1-3; Wilcox group, Eocene, Alabama.Toulmin, 1941, Jour. Paleontology, vol. 15, p. 584, pl. 79, fig. 13; Salt Mountain limestone, Alabama.

Test elongate, slightly tapered, almost straight; sutures somewhat indistinct, inclined to axis; average diameter, $0.13 \mathrm{~mm}$.; length ( 5 chambers), $0.39 \mathrm{~mm}$.

This fragment is referred to this common Wilcox species with some doubt. It is not recorded in the Midway of Texas or Alabama.

Dentalina cf. D. Cooperensis Cushman Plate 54, figure 21

Dentalina cooperensis Cushman, 1933, Cushman Lab. Foram. Research Contr., vol. 9, p. 8, pl. 1, fig. 17; Upper Jackson, Eocene, southeastern Coastal Plain.-Cushman, 1935, U. S. Geol. Survey Prof. Paper 181, p. 20, pl. 8, figs. 3, 4; Eocene, Southeastern United States.

Test elongate, compressed, somewhat tapered, gently curved, apical end slightly pointed; sutures distinct, only very slightly oblique to axis; wall smooth; apical end pointed; diameter, $0.10 \mathrm{~mm}$., length (6 chambers), $0.45 \mathrm{~mm}$. 
This specimen, like that of $D$. communis, is of doubtful affinity and is questionably referred to this species.

\section{Dentalia cf. D. pauperata d'Orbigny Plate 54, figure 10}

Dentalina pauperata d'Orbigny, 1846, Foram. Foss. Vienne, p. 46, pl. 1, figs. 57, 58.-Bournemann, 1855, Zeit. deutsch. geol. Gesell., vol. 7, p. 324, pl. 13, fig. 7.- Sherborn and Chapman, 1886, Roy. Micr. Soc. Jour., p. 750, pl. 15, fig. 9.-Cushman, 1940, Cushman Lab. Foram. Research Contr., vol. 16, p. 58, pl. 10, fig. 15; Midway group, Paleocene, Alabama.

Nodosaria pauperata, H. B. Brady, 1884, Challenger, vol. 9 (zool.), p. 500, text fig. 14.Chapman, 1893, Roy, Micr. Soc. Jour., p. 588, pl. 8, fig. 32.-Cushman, 1913, U. S. Nat. Mus. Bull. 71, pt. 3, p. 51, pl. 25, fig. 7.Halkyard, 1917, Proc. Manchester Lit. Phil. Soc. Mem., vol. 62, no. 6, p. 71 , pl. 4, figs. 8, 9.-Cushman, 1923, U. S. Nat. Mus. Bull. 104, pt. 4, p. 72, pl. 14, fig. 13.-Plummer, 1927, Texas Univ. Bull. 2644, p. 79, pl. 4, fig. 11; Midway group, Paleocene, Texas.

Test elongate, slightly tapered; chambers slightly arcuate, short, wide; initial chamber narrower, longer than those above, with terminal spine; walls smooth; sutures fairly prominent, transverse; average diameter, $0.33 \mathrm{~mm}$.

This long ranged species, abundant in the Midway of Texas and Alabama, has no value as a marker.

Dentalina spira Cooper, n. sp. Plate 54, figures 1,2

Chambers globular, almost spherical; surface marked by 14 wide prominent ribs, some of which may not continue over entire length of chamber, tapering and merging with shell surface near sutures; somewhat spiral in arrangement when viewed from above; diameter, $1.25 \mathrm{~mm}$; height (one chamber), $1.2 \mathrm{~mm}$.

D. spira does not appear to conform to any known species of Dentalina in the character and spiral arrangement of the ribs.

\section{Genus LaGena Walker and Jacob LAGENa LAEvis (Montagu) Plate 54, figures 6, 7}

Serpula (Lagena) laevis ovalis Walker and Boys, 1784, Test. Min. p. 3, pl. 1, fig. 9.

Vermiculum laeve, Montasu, 1803, Test. Brit., p. 524.

Lagena laevis, .Cushman, 1913, U. S. Nat. Mus. Bull. 71, pt. 3, p. 5, pl. 1, fig. 3, pl. 38, fig. 5; Recent, Pacific.-Howe, 1939, Louisiana Dept. Cons. Geol. Bull. 14, p. 50, pl. 6, fig. 12; Claiborne group, Eocene, Louisiana.-Toulmin, 1941, Jour. Paleontology, vol. 15, p. 593, pl. 80, fig. 7; Salt Mountain limestone, Eocene, Alabama.

Test almost globular, slightly elliptical in lateral view; neck cylindrical, set abruptly on chamber; wall smooth; diameter 0.40 $\mathrm{mm}$., length, probably $0.60-0.70 \mathrm{~mm}$.

Although not known from the Cretaceous, this species ranges throughout the Tertiary.

\section{Genus Nodosaria Lamark Nodosaria latejugata Gümbel Plate 55, figures 24, 25}

Nodosaria latejugata Gumbel, 1870, K. bayer. Akad. Wiss. München, Cl. 2, Abh., vol. 10,

\section{Explanation of Plate 54}

Figs. 1, 2-Dentalina spira Cooper, n. sp. 1, Sideview; 2, top view, $\times 20$.

3-5-Anomalina acuta Plummer. 3, Ventral view; 4 , peripheral view; 5 , dorsal view, $\times 90$.

6, 7-Lagena laevis (Montagu). 6, Apertural view; 7, lateral view, $\times 60$.

8, 9-Globigerina compressa Plummer. 8, Ventral view; dorsal view, $\times 90$.

10-Dentalina cf. D. pauperata d'Orbigny, $\times 90$.

11-Nodosaria spinocostata Cooper, n. sp., $\times 60$.

12, 13-Globigerina triloculinoides Plummer, $\times 90$

$14-$ Siphogeneroides? eleganta Plummer, $\times 90$. view, $\times 90$.

18, 19-Ellipsonodosaria plummerae Cushman. $\times 90$.

20 - Ellipsonodosaria spinulosa (Plummer). $\times 90$.

(p. 354)

(p. 352)

21-Dentalina $\mathrm{cf}$. D. cooperensis Cushman. $\times 90$.

(p. 352)

22 - Ellipsonodosaria saginensis (Plummer). $\times 90$.

(p. 347)

23-Ellipsonodosaria lepidula (Schwager). $\times 90$.

24, 25-Cibicides alleni (Plummer). 24, Ventral view; 25, dorsal view. $\times 60$. 


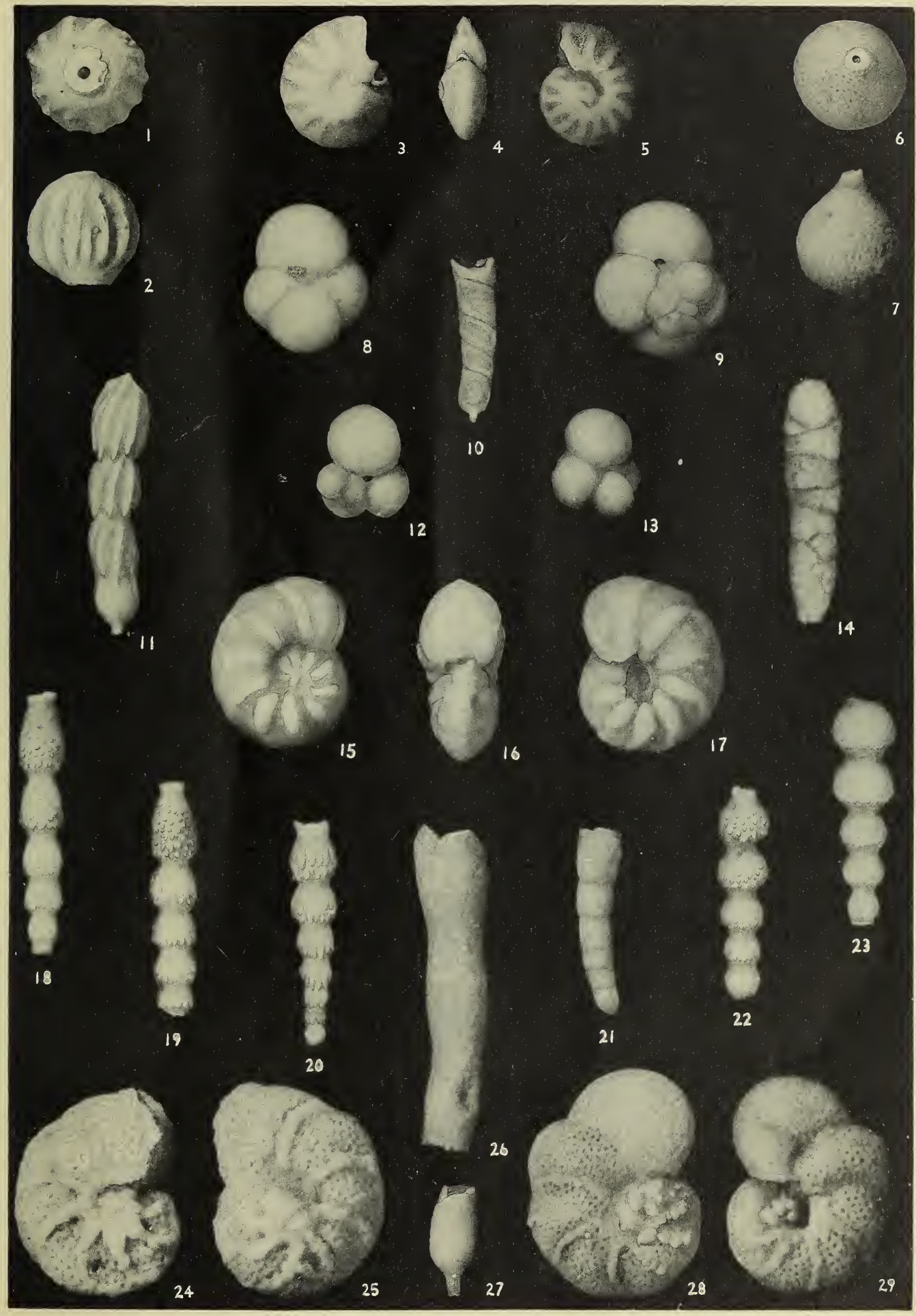

Cooper-Paleocene Foraminifera 


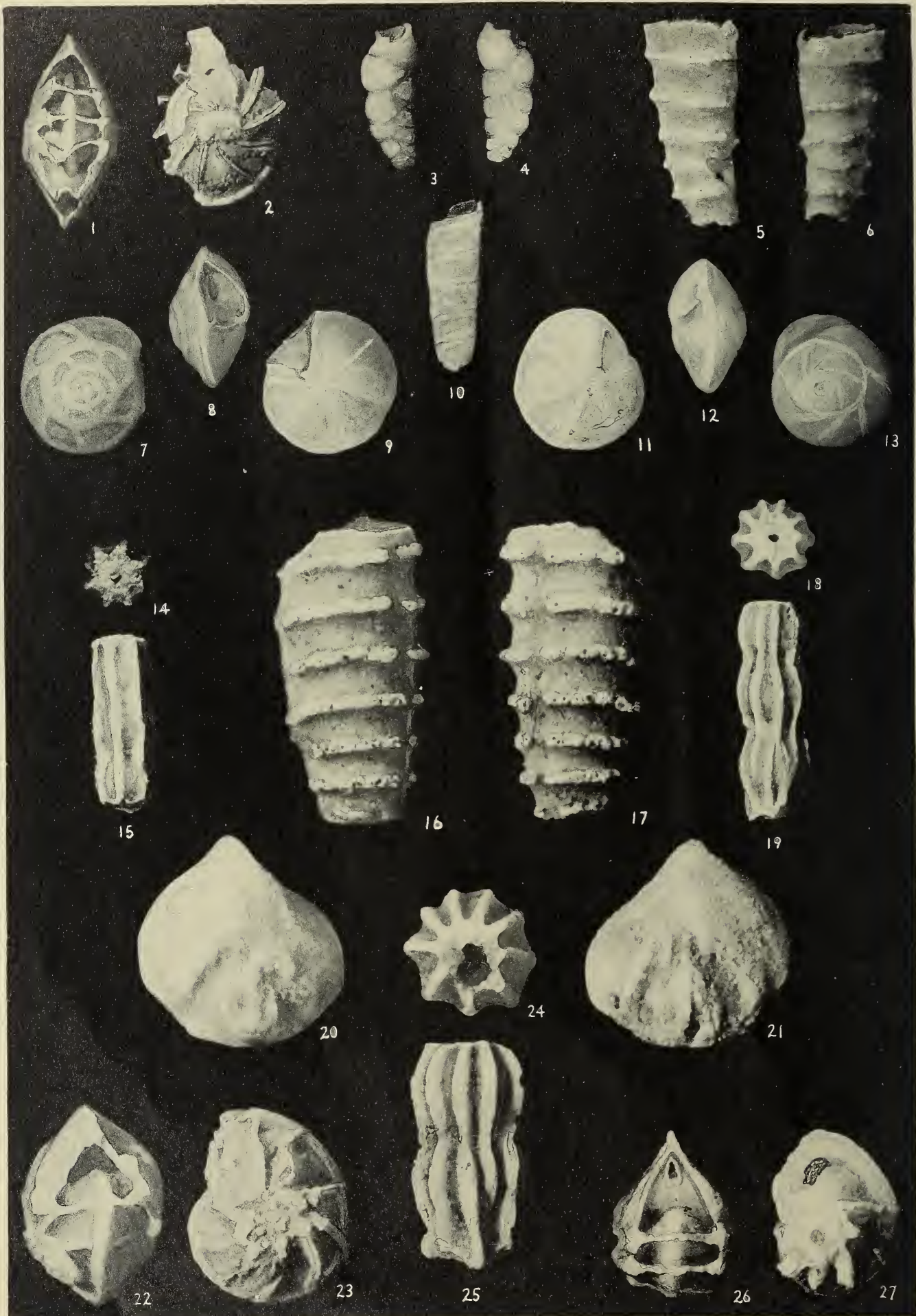

Cooper-Paleocene Foraminifera 
p. 619 , pl. 1, fig. 32; Upper Eocene, Bavaria.Hankten, 1876, Magy. kir. fäldt. int. Êvköuyoe, vol. 4, p. 21, pl. 2, figs. 6a-d; Eocene, Alabama.-Cushman, 1925, Cushman Lab. Foram. Research Contr., vol. 1, p. 66, pl. 10, fig. 7; Eocene, Alabama.-Cushman and Hanna, 1927, San Diego Soc. Nat. Hist. Trans., vol. 5, no. 4, p. 52, pl. 5, figs. 1-3; La Jolla formation, Eocene, California.-Cushman, 1935, U. S. Geol. Survey Prof. Paper 181, p. 21; Upper Eocene, U. S. Coastal Plain.-Cushman and McMasters, 1936, Jour. Paleontology, vol. 10 , p. 512, pl. 75, figs. 11, 12; Middle Eocene, California.-Toulmin, 1941, Jour. Paleontology, vol. 15, p. 588, pl. 79, figs. 26, 27; Salt Mountain limestone, Eocene, Alabama.

Nodosaria affinis Plummer, 1927, Texas Univ. Bull. 2644, p. 89, pl. 14, figs. 2a-d; Midway group, Paleocene, Texas.-Cushman, 1940, Cushman Lab. Foram. Research Contr., vol. 16 , p. 59, pl. 10, figs. 30-33; Midway group, Paleocene, Alabama.

Nodosaria burdensis Hankten, 1876, Magy. kir. fäldt. int. Évköuyoe, vol. 4, p. 23, pl. 2, fig. 10, pl. 16, fig. 4.

Nodosaria vertebralis Nuttall, 1932, Jour. Paleontology, vol. 6, p. 15, pl. 3, fig. 9; Alazan shale, Lower Oligocene, Tampico Embayment, Mexico.

Nodosaria sp. A, Israelsky, 1939, Sixth Pacific Sci. Cong. Proc. vol. 2, p. 575, pl. 3, fig. 11; Marysville formation, Eocene, California.

Test straight; chambers broad, only slightly inflated; with 9 strong longitudinal costate which continue across sutures and are normal to surface of chamber; sutures transverse; diameter $1.2 \mathrm{~mm}$.; length (2 chambers), $2.0 \mathrm{~mm}$.

This very strongly ribbed form seems quite variable in its specific characters. The fragmentary specimen figures represents one of the largest assigned to this species although it has the minimum number of costae. It is very common in Eocene sediments, especially in the lower formations. While quite similar to the Cretaceous $N$. zippei Reuss it differs from $N$. affinis Reuss, which has according to the original description only 4 to 5 costae.

\section{Nodosaria spinocostata Cooper, n. sp. Plate 54, figure 11}

Nodosaria spinulosa Plummer (part), 1927, Texas Univ. Bull. 2644, p. 84, pl. 4, fig. 19c only; Midway group, Paleocene, Texas.

Ellipsonodosaria alexanderi, Cushman (part), 1940, Cushman Lab. Foram. Research Contr. vol. 16 , p. 69 , pl. 11, figs. 27, 29 only; Midway group, Paleocene, Texas.

Test elongate, slightly curved; chambers elongate to globular, increasing in size and length upward; initial chamber smaller than one next above, free of costae, terminated by relatively large spine; costae on succeeding chambers nine or less, discontinuous, each terminating in spine overhanging constricted region of sutures; average diameter, $0.20 \mathrm{~mm}$.; length ( 3 chambers), $0.75 \mathrm{~mm}$.

This species differs from $N$. spinulosa (Montagu) of various authors and $E$. alexanderi Cushman in that it is regularly costate as in $N$. latejugata Gümbel and others of this type, but the costae of $N$. spinocostata end in spines as shown by Plummer (1927, p. 4, fig. 19c) and Cushman (1940, pl. 11, figs. 27, 29). The holotype of $E$. alexanderi Cushman (1936, pl. 9, fig. 9) shows the characteristically irregular distribution of the spines over the entire surface of the chamber wall.

\section{Explanation of Plate 55}

FIgs. 1,2-Robulus magnificus Toulmin. 1, Peripheral view ; 2, dorsal view. $\times 20$.

3, 4-Rectogïmbelina alabamensis Cushman. $\times 90$.

5, 6-Vaginulina gracilis Plummer. $\times 60$.

7-9_-Siphonina prima Plummer. 7 Dorsal view; 8, peripheral view; 9, ventral view. $\times 90$

10-Dentalina cf. D. communis (d'Orbigny). $\times 90$.

11-13-Eponides exigna limbata Plummer. 11, Ventral view; 12, peripheral view; 13 , dorsal view. $\times 90$.

(p. 353)

14, 15-Nodosaria cf. N. vertebralis (Batsch). 1, Top view of broken specimen; 2 , lateral view of three chambers. $\times 20$.

16, 17-Vaginulina robusta Plummer. Opposite sides of one specimen. $\times 40$.

$18,19-$ Nodosaria zippei Reuss. Top and lateral views. $\times 20$.

20, 21-Palmula mcglamerae Toulmin. Opposite views of one specimen. $\times 40$.

22-23-Robulus midwayensis (Plummer). 22, Peripheral view; 23, dorsal view. $\times 40$. (p. 351)

24-25-Nodosaria latejugata Gümbel. 24, Top view, broken along suture; 25 , lateral view. $\times 20$.

26-27-Robulus inornatus (d'Orbigny). 26, Peripheral view of broken shell showing the oval, slit-like aperature; 27 , dorsal view. $\times 40$. 
Nodosaria cf. N. vertebralis (Batsch) Plate 55, figures 14,15

Nautilus (Orthoceras) vertebralis Batsch, 1791, Conch. des Seesandes no. 6, p. 3, pl. 2, fig. 6; Recent, Mediterranean.

Nodosaria vertebralis, H. B. Brady, 1884, Challenger, vol. 9 (Zool.), p. 514, pl. 63, fig. 35; pl. 64, figs. 11-14; Recent.-Flint, 1899, U. S. Nat. Mus. Ann. Rept. for 1897 , p. 312, pl. 57, fig. 5; Recent.-Bagg, 1912, U. S. Geol. Survey Bull. 513 , p. 60 , pl. 17 , fig. 2 ; Pliocene and Pleistocene, California.-Cushman, 1913, U. S. Nat. Mus. Bull. 71 , pt. 3, p. 60, pl. 32, fig. 1; Recent, North Pacific.-Cushman, 1919, Carnegie Inst. Washington Pub. 291, p. 35, pl. 7, figs. 3-5; Recent, Caribbean and tropical Pacific, West Indies.-Cushman, 1919, U. S. Nat. Mus. Bull. 100, vol. 4, p. 211, pl. 38, figs. 2, 3; pl. 40, fig. 2; Recent, Philippine Archipelago.-Cushman, 1923, idem., Bull. 104, pt. 4, p. 86 , pl. 14, fig. 6; Recent, Gulf of Mexico.Plummer, 1927, Texas Univ. Bull. 2644, p. 88, pl. 5, fig. 10; Midway group, Paleocene, Texas.

This fragment showing three chambers seems closest to $N$. vertebralis by reason of the few continuous ribs and the almost total lack of inflation of the valves. It is common in the lower Midway of Texas but the species is known to range from the Cretaceous to Recent.

\section{Nodosaria cf. N. zIPPEI Reuss Plate 55, figures 18, 19}

Nodosaria zippei Reuss, 1844, Geogn. Skizze aus Böhmer, p. 210.-Reuss, 1845, Die Versteinerungen der böhemischen Kreide, pt. 1, p. 25, pl. 8, figs. 1-3; Cretaceous, Bohemia.Beissel and Halzapfel, 1891, Abh. d. geol. Landesanstalt, n. ser., no. 3, pt. 6, pp. 31, 32, figs. 10-29, pl. 16, fig. 32.-Egger, 1899, Abh. d. II, Ck. d. k. Ak. d. Wiss, vol. 21, pt. 1, p. 78, pl. 8, fig. 3.-Plummer, 1931, Texas Univ. Bull. 3101, p. 157; Cretaceous, Texas.Sandidge, 1932, Jour. Paleontology, vol. 6, p. 275, pl. 42, figs. 13, 14; Ripley formation, Cretaceous, Texas.-Jennings, 1936, Bull. Am. Paleontology, vol. 23, p. 178, pl. 2, fig. 13 Navisink formation, Cretaceous, New Jersey.

Test elongate, straight; chambers numerous; globular, separated by prominent, deeply incised, transverse sutures; ribs 9, raised, fin-like, rarely fused, more frequen tly abruptly terminated; diameter, $0.8 \mathrm{~mm}$.; length ( 2 chambers), $0.17 \mathrm{~mm}$.

The specimen illustrated has been referred to this species because of the joining or fusing of some costae and the termination of others; both features are shown in figure 19. This specimen is classified with
N. zippei with some hesitancy because all other described forms are from the Cretaceous.

Genus Palmula Lea

Palmula mcglameryae Toulmin Plate 55, figures 20, 21

Palmula mcglameryae Toulmin, 1941, Jour. Palaeontology, vol. 15 , p. 592 , pl. 80 , figs. $1-3$; Salt Mountain limestone, Eocene, Alabama. Polymorphina cushmani Plummer, 1927, Texas Univ. Bull. 2644, p. 125, pl. 6, fig. 9.

Test wide, compressed, thickest through rounded base; periphery irregular or asymmetric, rounded on basal margin, produced to point on aperatural end; width, $1.1 \mathrm{~mm}$.; height, $1.2 \mathrm{~mm}$.

Although the distal end is more rounded than the form illustrated by Plummer, Toul$\min (1941)$ in his three figures and in his remarks demonstrates the great variability of the outline of this species.

\section{Genus Robulus Montfort \\ Robulus inORnatus (d'Orbigny) Plate 55, figures 26, 27}

Robulina inornata d'Orbigny, 1846, Foram. Foss. Bass. Tert. Vienne, p. 102, pl. 4, figs. 25, 26; Miocene, Austria.

Cristellaria inornata, Cushman and Hanna, 1927, California Acad. Sci. Proc., ser. 4, vol. 16, p. 217, pl. 14, fig. 5; Eocene, California.-Nuttall, 1932, Jour. Paleontology, vol. 6, p. 10; Eocene, Mexico.-Palmer and Bermúdez, 1936, Soc. Cubana Hist. Nat. Mem., vol. 10, no. 4, p. 249; Oligocene, Cuba.

Robulus inornatus, Cushman and Backsdale, 1930, Stanford Univ. Dept. Geol. Contr., vol. 1, no. 2, p. 62, pl. 11, figs. 2, 3; Eocene, California.-?Israelsky, 1939, Proc. Sixth Pacific Sci. Cong., p. 573, pl. 3, fig. 1; Marysville formation (Capay stage), Eocene, California.Toulmin, 1941, Jour. Paleontology, vol. 15, p. 577 , pl. 78 , fig. 19, text fig. 2B; Salt Mountain limestone, Eocene, Alabama.

Test large, somewhat compressed, thickness three-fourths of diameter, closely coiled, periphery acutely keeled; chambers 7 in last whorl, triangular in outline, increasing rapidly in size to final one; sutures distinctly limbate, slightly curved, tangent to large umbo; diameter, $1.0 \mathrm{~mm}$; thickness, 0.75 $\mathrm{mm}$.

\section{Robulus magnificus Toulmin} Plate 55, figures 1, 2

Robulus magnificus Toulmin, 1941, Jour. Paleontology, vol. 15 , p. 578 , pl. 78 , fig. 22 , text fig. 
2D; Salt Mountain limestone, Eocene, Alabama.

Test compressed, thickness about onehalf of diameter; periphery distinctly keeled in early chambers, less so in final ones; chambers 9 in last whorl; sutures almost straight to slightly curved, raised, distinctly limbate, subtangent to, but not meeting umbo in final two or three chambers; diameter, $2.0 \mathrm{~mm}$.; thickness, $1.1 \mathrm{~mm}$.

This form seems identical with $R$. magnificus described by Toulmin from Alabama, except that the sutures of the Illinois specimen are somewhat less curved.

\section{Robulus midwayensis (Plummer) Plate 55, figures 22, 23}

Cristellaria midwayensis Plummer, 1927, Texas Univ. Bull. 2644, p. 95, pl. 13, figs. 5a-c; Midway group. Paleocene, Texas.

Lenticulina midwayana Israelsky, 1939, Proc. Sixth Pacific Sci. Cong., p. 573, pl. 2, figs. 7, 8; Marysville formation (Capay stage), Eocene, California.

Robulus midwayensis Cushman, 1940, Cushman Lab. Foram. Research Contr., vol. 16, p. 54, pl. 9, fig. 12; Midway group, Paleocene, Alabama.-Toulmin, 1941, Jour. Paleontology, vol. 15 , p. 579 , pl. 78 , fig. 23 , text fig. $2 \mathrm{G}$; Salt Mountain limestone, Eocene, Alabama.

Test large, only slightly compressed, dia meter and thickness about equal; chambers 9 in last whorl; slightly curved, almost equal in size, increasing very gradually to final chamber; sutures prominent, limbate, tapering, with greatest curvature near peripheral margin; diameter and thickness, about 1.2 $\mathrm{mm}$.

The Illinois specimen is nodose and thus resembles the Texas form but Salt Mountain specimens have a smooth umbo. This species is a good Midway marker in Texas.

\section{Genus Vaginulina d'Orbigny VAGINUlina GRacilis Plummer Plate 55, figures 5, 6}

Vagulina gracilis Plummer, 1927, Texas Univ. Bull. 2644, p. 111, pl. 6, figs. 5a-b; Midway group, Paleocene, Texas.-Cushman, 1940, Cushman Lab. Foram. Research Contr., vol. 16, p. 61, pl. 9, fig. 27; Midway group, Paleocene, Alabama.

Test tapering, slightly arcuate, compressed; chambers short; sutures only slightly oblique, marked by abrupt, narrow, somewhat nodose and discontinuous ridges; diameter, average, $0.3 \mathrm{~mm}$; height, 5 chambers, $0.50 \mathrm{~mm}$.

This species is common in the lower Midway of Alabama and Texas, where it is succeeded in the upper Midway by $V$. robusta Plummer.

\section{Vaginulina Robusta Plummer Plate 55, figures 16, 17}

Vaginulina robusta Plummer, 1927, Texas Univ. Bull. 2644 , p. 112 , pl. 6, figs. $4 a-b$, pl. 13 , fig. 3; Midway group, Paleocene, Texas.-Cushman, 1940, Cushman Lab. Foram. Research Contr., vol. 16, p. 61, pl. 10, figs. 1-4; Midway group, Paleocene, Alabama.

Test, large, robust, slightly compressed; straight; chambers smooth, short but quite broad; sutures transverse to slightly oblique, prominent, somewhat nodose; nodes discontinuous along more convex edges (in double row on broader edge, see figs. 16, 17); average diameter, $0.8 \mathrm{~mm}$; height, 5 chambers, $1.3 \mathrm{~mm}$.

This species is characteristic of the upper Midway in Texas and Alabama. It is straighter and more robust than $V$. gracilis.

Family Heterohelicidae Cushman

Genus Rectogümbelina Cushman

Rectogümbelina alabamensis Cushman Plate 55, figures 3,4

Rectogïmbelina alabamensis Cushman, 1940, Cushman Lab. Foram. Research Contr., vol. 16, p. 65, pl. 11, fig. 16; Midway group, Paleocene, Alabama.

Test very small, slightly arcuate, slender, tapering; early chambers biserial, later ones uniserial, biserial chambers less inflated; early sutures slightly and less depressed than later ones; diameter $0.065-0.145 \mathrm{~mm}$.; length, 0.37 .

Although slightly larger than the form described by Cushman from Alabama, the Illinois specimen seems to conform in all other respects. Common in the upper Midway of Alabama.

Genus Siphogeneroides Cushman

Siphogeneroides? Eleganta (Plummer) Plate 54, figure 14

Siphogenerina eleganta Plummer, 1927, Texas Univ. Bull. 2644, p. 126, pl. 8, figs. 1a-c; Midway group, Paleocene, Texas.-Cushman, 1940, Cushman Lab. Foram. Research Contr., vol. 16, p. 66, pl. 11, fig. 17; Midway group, Paleo. cene, Alabama. 
Test small, elongate, straight, tapering; sarly chambers indistinct, may be bi- or triserial, later chambers irregular in size and shape, arranged alternately in oblique manner; sutures distinct, sharply incised; aperature terminal; diameter, $0.072-0.15 \mathrm{~mm}$.; length, $0.50 \mathrm{~mm}$.

This species, which is placed in synonymy with Siphogenerina eleganta Plummer by Cushman, is described as biserial by Plummer and triserial by Cushman. The early chambers of the Illinois specimen are too indistinct for accurate determination. It is common in the upper Midway of Texas and the lower Midway of Alabama.

\section{Family Ellipsoidinidae}

Genus Ellipsonodosaria A. Silvestri

Ellipsonodosaria lePidula (Schwager)

Plate 54, figure 23

Nodosaria lepidula Schwager, 1866, NovaraExped. Geol. Theil., vol. 2, p. 210, pl. 5, figs. 27-28; Pliocene.-Galloway and Morey, 1929, Bull. Am. Paleontology, vol. 15 , no. 55, p. 17, pl. 2, fig. 2; Eocene, Ecuador-Galloway and Morey, 1931, Jour. Paleontology, vol. 5, p. 337, pl. 38, fig. 1, Upper Cretaceous, Mexico.

Ellipsonodosaria sp. Cushman, 1940, Cushman

Lab. Foram. Research Contr., vol. 16, p. 70,

pl. 12, figs. 1, 2; Midway group, Paleocene, Alabama.

Test elongate, tapering, straight; chambers globular, smooth except that for lower margin is angular, fimbriate; diameter, 0.078-0.17 mm.; length, 5 chambers, 0.55 $\mathrm{mm}$.

E. lepidula is distinguished by the globular, almost spherical chambers, which are connected by short "necks," and which are smooth except for a very narrow fimbriate margin on the lower edge. Rare in the late Cretaceous of Mexico and the lower Midway of Alabama.

\section{Ellipsonodosaria plummerae Cushman Plate 54, figures 18, 19}

Ellipsonodosaria plummerae Cushman, 1940, Cushman Lab. Foram. Research Contr., vol. 16 , p. 69 , pl. 12, figs. 4, 5; Midway group, Pliocene, Texas.

Test elongate, somewhat tapering, straight; chambers pyriform, distinct, increasing in size and becoming more pyriform upward, very spinose, especially at ridge near base of chamber; aperture ter- minal, rounded, phialine; diameter, 0.078 $0.1 .3 \mathrm{~mm}$., length, 5 chambers, $0.61 \mathrm{~mm}$.

This species seems very close to $E$. pseudoscripta Cushman from the Upper Cretaceous and to E. sagrinensis (Bagg) from the Midway. It differs from the latter, however, in its more elongate chambers and from the former by the more abrupt margins on the lower edges of the chambers.

\section{Ellipsonodosaria SAgRinensis (Bagg)} Plate 54, figure 22

Nodosaria sagrinensis Bagg, 1912, U. S. Geol. Survey Bull. 513, p. 58, pl. 16, fig. 4; Pliocene, California.-Plummer, 1927, Texas Univ. Bull. 2644, p. 85, pl. 4, fig. 16; Midway group, Paleocene, Texas.

Nodogenerina sagrinensis Jennings, 1936, Bull.

Am. Paleontology, vol. 23, p. 187, pl. 3, fig. 17;

Hornerstown, Eocene, New Jersey.

Test straight, tapered; chambers short to globular in early stages, becoming more elongate later, lower edge fimbriate, upper part narrowing toward sutures, wall thickly spinose; spines short above, longer below, overhanging lower edge; sutures deep, broad; average diameter, $0.10 \mathrm{~mm}$.; length, 5 chambers, $0.50 \mathrm{~mm}$.

This species was placed in synonymy with E. plummerae by Cushman (1940, p. 69) but its chambers do not seem to be sufficiently elongate to warrant its reference to that species. It is abundant in the upper, but rare to common in the lower Midway of Texas, and has also been reported from the Navarro.

\section{Ellipsonodosaria spinulosa (Plummer) Plate 54, figure 20}

Nodosaria spinulosa (part) Plummer, 1927, Texas Univ. Bull. 2644, p. 85, pl. 4, fig. 19a, b only; Midway, Paleocene, Texas.

Test elongate, tapering, straight to slightly arcuate; chambers somewhat elongate, covered with spine-terminated ribs; sutures depressed; average diameter, 0.10 $\mathrm{mm}$.; height, 7 chambers, $0.50 \mathrm{~mm}$.

This form differs from Nodosaria spinocostata n. sp. and from N. spinulosa Plummer (1927, pl. 4, fig. 19c), in that its ribs may terminate in spines over the entire chamber wall while in $N$. spinocostata the ribs occupy the entire length of the chamber wall and terminate in a spine overhanging the sutural depression. 


\section{Family Rotalindae Reuss \\ Genus Eponides Montfort \\ EPONIDES EXIGUA LIMBata (Plummer) \\ Plate 55, figures 11-13}

Pulvinulina exigua limbata Plummer, 1927, Texas Univ. Bull. 2644, p. 152, pl. 11, figs. 4a-c; Midway group, Paleocene, Texas.

?Eponides sp. Cushman, 1940, Cushman Lab. Foram. Research Contr., vol. 16, p. 71, pl. 12, figs. 8a, b; Midway group, Paleocene, Alabama.

Test small, biconvex, peripheral margin sharp or acute, indistinctly lobed, chambers 5 in final whorl, smooth; sutures very indistinct, on dorsal side oblique, straight, narrow; ventral sutures flush, almost straight but slightly curved from umbilicus; diameter, $0.30 \mathrm{~mm}$.; thickness, $0.20 \mathrm{~mm}$.

This species and its two varieties are common in the Midway of Texas. The variety limbata is common in the basal strata and var. exigua forms a good marker for the lower beds. The Alabama form is limited to the upper Midway.

\section{Genus Siphonina Reuss \\ Siphonina PRIMA Plummer \\ Plate 55, figures 7-9}

Siphonina prima Plummer, 1927, Texas Univ. Bull. 2644, p. 148, pl. 12, figs. 4a-c; Midway group, Paleocene, Texas.-Cushman, 1927, U. S. Nat. Mus. Proc., vol. 72, art. 20, p. 2, pl. 2, figs. 4a-c.-Cushman, 1940, Cushman Lab. Foran. Research Contr., vol. 16, p. 71, pl. 12, fig. 10; Midway group, Paleocene, Alabama; Navarro, Cretaceous, Gulf Coast Embayment.

Test circular, biconvex; peripheral angle acute; margin lobate, chambers 5 in last whorl; dorsal sutures curved, broad; ventral sutures straight, narrow, excavated, radiate from shallow central depressed area; diameter, $0.35 \mathrm{~mm}$.; thickness, $0.20 \mathrm{~mm}$.

It is common in the upper Midway of Texas, Alabama, Arkansas, Mississippi, and Tennessee, but is also present in the Upper Cretaceous (Navarro) in these states.

\section{Family Globigerinidae Carpenter Genus Globigerina d'Orbigny Globigerina compressa Plummer Plate 54, figures 8, 9}

Globigerina compressa Plummer, 1927, Texas Univ. Bull. 2644, p. 135, pl. 8, figs. $11 \mathrm{a}-\mathrm{c}$; Midway group, Paleocene, Texas.-Jennings, 1936, Bull. Am. Paleontology, vol. 23, p. 193. pl. 31, fig. 8; Hornerstown formation, Eocene, New Jersey.-Toulmin, 1941, Jour. Paleontology, vol. 15, p. 607, pl. 82, figs. 1, 2; Salt Mountain limestone, Eocene, Alabama.

Test small, quadrate, closely coiled, compressed, margin lobate; chambers increasing regularly, inflated, overlapping dorsally; sutures strongly depressed; wall thin, smooth, finely punctate; single aperature arched; diameter, 0.30 to $0.40 \mathrm{~mm}$.

It is common in the upper Midway of Texas.

Globigerina triloculinoides Plummer Plate 54, figures 12, 13

Globigerina triloculinoides Plummer, 1927, Texas Univ. Bull. 2644, p. 134, pl. 8, figs. 10a-c; Midway group, Paleocene, Texas.-Jennings, 1936, Bull. Am. Paleontology, vol. 23, p. 193, pl. 31, fig. 10; Hornerstown formation, Eocene, New Jersey.-Glaessner, 1937, Moscow Univ. Publ. Lab. Paleontology, vols. 2-3, p. 382, pl. 4, figs. 33a, b; Lower Tertiary, Caucasus.Cushman, 1940, Cushman Lab. Foram. Research Contr., vol. 16, p. 72, pl. 12, fig. 15; Midway group, Paleocene, Alabama.-Toulmin, 1941, Jour. Paleontology, vol. 15, p. 607, pl. 82, fig. 3; Salt Mountain limestone, Eocene, Alabama.

Test very small, trochoid, periphery distinctly lobate; last volution formed by 3 large globular chambers which increase rapidly in size; surface finely punctate; diameter, 0.25 to $0.30 \mathrm{~mm}$.

It is common in the upper Midway of Texas and Alabama and the Salt Mountain limestone of the Alabama Wilcox.

\section{Family Anomalinidae Cushman Genus Anomalina d'Orbigny Anomalina acuta Plummer Plate 54, figures 3-5}

Anomalina ammonoides acuta Plummer, 1927, Texas Univ. Bull. 2644, p. 149, pl. 10, figs. 2a-c; Midway group, Paleocene, Texas.

Anomalina acuta, Glaessner, 1937, Moscow Publ. Lab. Paleontology, vols. 2-3, p. 386, pl. 5, figs. 39a-c. Lower Tertiary, Caucasus.-Toulmin, 1941, Jour. Paleontology, vol. 15, p. 608, pl. 82, figs. 9, 10; Salt Mountain limestone (lower Wilcox).

Test compressed, involute, about equally biconvex, periphery acute; chambers narrow, numerous, 13 in last whorl; dorsal sutures distinct, limbate to slightly depressed; ventral sutures thickened around umbilical area, merging into prominently developed 
or elevated central boss; shell perforate; diameter, $0.31 \mathrm{~mm}$.; thickness, $0.12 \mathrm{~mm}$.

This species is common in the two faunal zones of the Midway of Texas and serves to distinguish these horizons from the underlying Cretaceous formations. It also occurs in the lower Wilcox of Alabama.

\section{Anomalina midwayensis (Plummer)} Plate 54, figures $15-17$

Truncatulina midwayensis Plummer, 1927, Texas Univ. Bull. 2644, p. 141, pl. 9, figs. 7a-c, pl. 15, figs. 3a-b; Midway group, Paleocene, Texas.

Anomalina midwayensis, Cushman, 1940, Cushman Lab. Foram. Research Contr., vol. 16, p. 73, pl. 12, fig. 18; Midway group, Paleocene, Texas.

Test biconvex, little compressed; chambers 9 to 10 in last whorl, punctate; sutures strongly elevated, broad near umbilicus but separated from it, tapering toward margin, curved; slit-like aperature at base of septal face; diameter, $0.41 \mathrm{~mm}$., thickness, 0.22 $\mathrm{mm}$.

This form is identical with the Texas Midway form in all respects except that it is somewhat thicker and the sutures a little wider, especially near the umbilicus. The species is found throughout the Midway of Texas and in the upper Midway of Alabama.

\section{Genus Cibicides Montfort Cibicides alleni (Plummer) Plate 54, figures 24, 25}

Truncatulina alleni Plummer, 1927, Texas Univ. Bull. 2644, p. 144, pl. 10, figs. 4a-c; Midway group, Paleocene, Texas.-Cushman, 1940, Cushman Lab. Foram. Research Contr., vol. 16 , p. 73, pl. 12, fig. 19; Midway group, Paleocene, Alabama.

Test unequally biconvex, more rounded on ventral side; peripheral margin slightly acute, somewhat lobate; chambers, 9? in last whorl, coarsely punctate; sutures and periphery conspicuously marked by shell material; sutures curved, gently tapering from large, smooth umbilicus; aperature large, arched, extending far down on ventral side; diameter, 0.60 to $0.80 \mathrm{~mm}$.

It is common in the middle and upper Midway of Texas and Alabama but is rare in the Navarro.

\section{Cibicides vulgaris (Plummer) Plate 54, figures 28, 29}

Truncatulina vulgaris Plummer, 1927, Texas Univ. Bull. 2644 , p. 145 , pl. 10 , figs. $3 \mathrm{a}-\mathrm{c}$; Midway group, Paleocene, Texas.-Cushman, 1940, Cushman Lab. Foram. Research Contr., vol. 16, p. 73, pl. 12, fig. 21; Midway group, Paleocene, Alabama.

Test unequally biconvex; periphery broadly rounded, lobate; chambers 8 in last whorl, later ones turgid; sutures curved, tapering, elevated by shell material, walls coarsely punctate; slit-like aperature extending from periphery to umbilicus; diameter, 0.60 to $0.85 \mathrm{~mm}$.

It is abundant in the upper and rare in the lower Midway of Alabama and Texas.

\section{REFERENCES}

Cushman, Joseph A., 1940, Midway Foraminifera from Alabama: Cushman Lab. Foram. Research Contr., vol. 16, pp. 51-73.

Farrar, Willard, and McManamy, Lyle, 1937, The geology of Stoddard County, Missouri: Missouri Geol. Survey, 59 Bien. Rept., App. 6, pp. 24-29.

HARris, G. D., 1896, The Midway stage: Bull. Am. Paleontology, vol. 1, no. 4, pp. 18-22.

Kansas Geol. Society Guidebook, 13th Ann. Field Conference, 1939.

Lamar, J. E., and Sutton, A. H., 1930, Cretaceous and Tertiary sediments of Kentucky, Illinois and Missouri: Am. Assoc. Petroleum Geologists Bull., vol. 14, pp. 845-866.

McFarlan, ARThUR C., 1943, Geology of Kentucky, Kentucky Univ.

Plummer, Helen J., 1927, Foraminifera of the Midway formation in Texas: Texas Univ. Bull. 2644.

Sardeson, F. W., 1898, The so-called Cretaceous deposits in southeastern Minnesota: Jour. Geology, vol. 16, pp. 679-691:

StafFord, J. M., 1864, On the Cretaceous and superior formations of west Tennessee: Am. Jour. Sci., ser. 2, vol. 37, pp. 360-372.

Stewart, Dan R., McManamy, LyLe, and MCQueEn, HeNRy S., 1943, Occurrence of bauxite clay in Stoddard County, Missouri: Missouri Geol. Survey, 62 Bien. Rept., App. III.

Stose, George W., 1932, Geologic map of the United States. U. S. Geol. Survey.

Toulmin, Lyman D., 1941, Eocene smaller Foraminifera from the Salt Mountain limestone of Alabama: Jour. Paleontology, vol. 15, pp. 567-611, pls. 78-82.

WELLER, J. MARVIN, 1939, Geologic map of Illinois: Illinois Geol. Survey, Blue line print $1: 500,000$.

Whitlatch, Geo. I., 1940, The clays of west Tennessee: Tennessee Geol. Survey, Bull. 49. 